Supporting Information

\title{
Measurement of Antioxidant Capacity by Electron Spin Resonance Spectroscopy Based on Copper (II) Reduction
}

Dan $\mathrm{Li}^{\mathrm{a}}$, Jia Jiang ${ }^{\mathrm{a}}$, Dandan Han ${ }^{\mathrm{b}}$, Xinyu Yu ${ }^{\mathrm{b}}$, Kun Wang ${ }^{\mathrm{a}}$, Shuang Zang ${ }^{\mathrm{a}}$, Dayong Lu ${ }^{\mathrm{b}}$, Aimin $\mathrm{Yu}^{\mathrm{a}}$, Ziwei Zhang ${ }^{\mathrm{a}}{ }^{\text {* }}$

${ }^{a}$ College of Chemistry, Jilin University, Qianjin Street 2699, Changchun 130012, PR

China

${ }^{b}$ Department of Materials Science and Engineering, Jilin Institute of Chemical Technology, Jilin 132022, PR China

Corresponding author: Ziwei Zhang

Tel.: +86 13610709861; fax: +86 43185112355

E-mail address: zzw@j]lu.edu.cn

\section{Supporting Figures}

Figure S1. Standard curves for the antioxidants obtained by ESR spectroscopy

Figure S2. Standard curves for the antioxidants obtained by UV spectrometry 

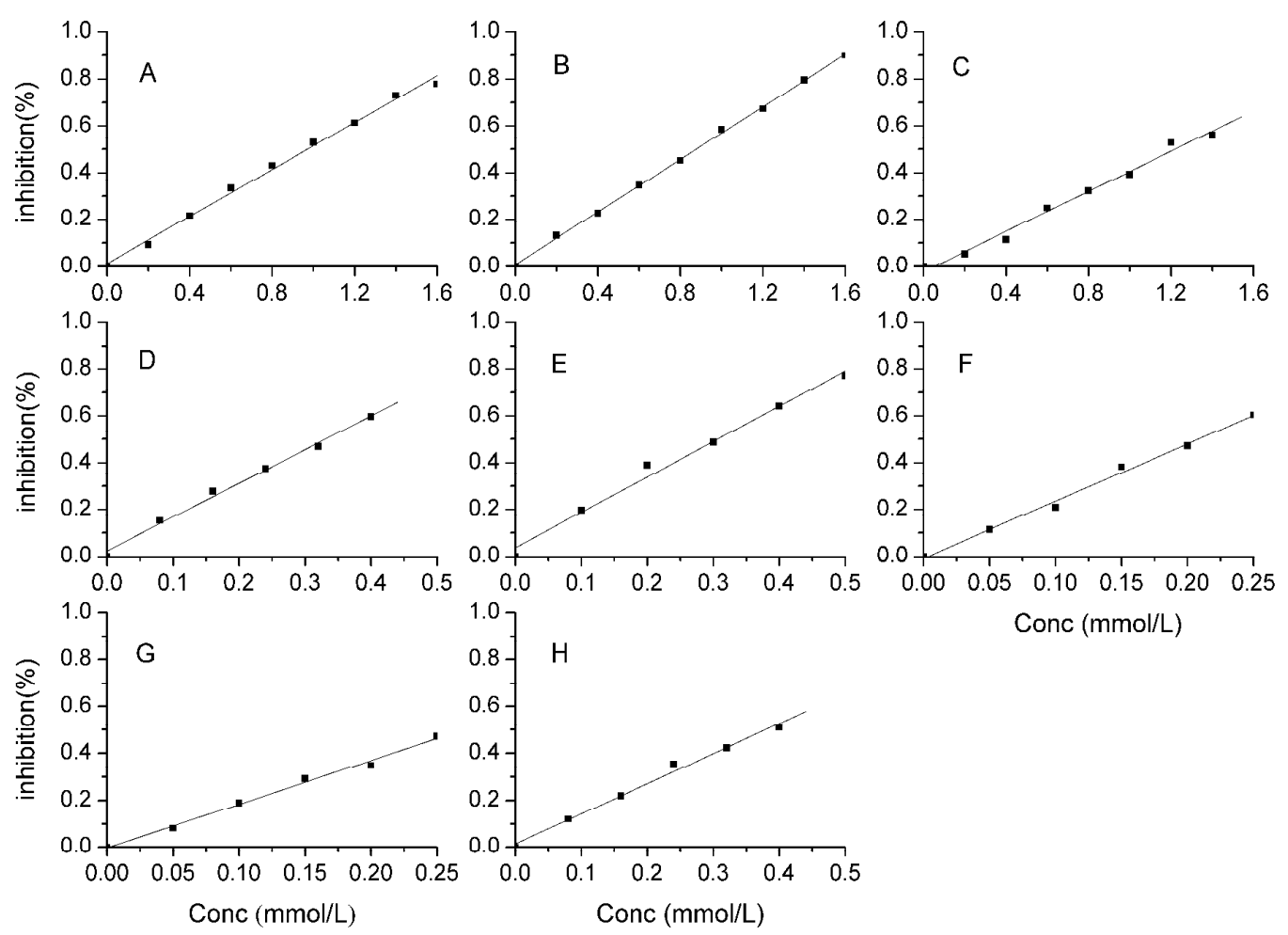

Conc $(\mathrm{mmol} / \mathrm{L})$

Figure S1. Standard curves for the antioxidants obtained by ESR spectroscopy. (A) Trolox; (B) ascorbic acid; (C) ferulic acid; (D) rutin; (E) caffeic acid; (F) quercetin; (G) chlorogenic acid; (H) gallic acid. 

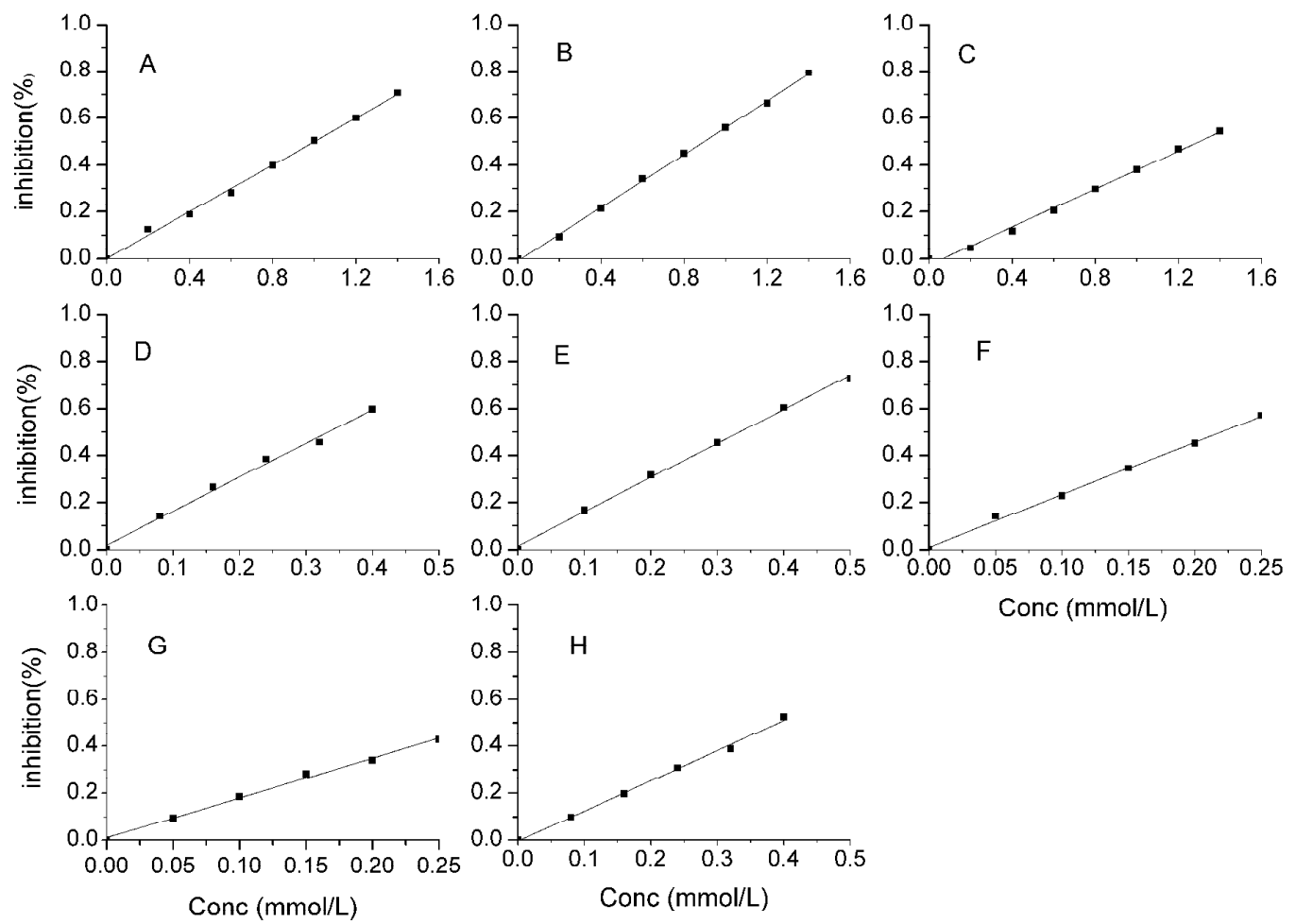

Conc (mmol/L)

Figure S2. Standard curves for the antioxidants obtained by UV spectrometry. (A) Trolox; (B) ascorbic acid; (C) ferulic acid; (D) rutin; (E) caffeic acid; (F) quercetin; (G) chlorogenic acid; (H) gallic acid. 